\title{
Ciclo reproductivo y fecundidad parcial de Isacia conceptionis (Perciformes, Haemulidae) en La Herradura, Coquimbo, Chile
}

Reproductive cycle and batch fecundity of Isacia conceptionis (Perciformes, Haemulidae) at La Herradura, Coquimbo, Chile

\section{Niris Cortés ${ }^{1}$ y Alejandro Aron ${ }^{2}$}

\author{
${ }^{1}$ Escuela de Prevención de Riesgos y Medio Ambiente, Facultad de Ciencias del Mar, Universidad Católica del Norte, Larrondo 1281, \\ Casilla 117, Coquimbo, Chile.ncortes@ucn.cl \\ ${ }^{2}$ Compañía Minera San Gerónimo. Av. Talca 101, Barrio Industrial Alto Peñuelas, Coquimbo, Chile
}

\begin{abstract}
This study analyses reproductive aspects of the fish Isacia conceptionis based on 280 females collected at La Herradura Bay, northern Chile, between April 1995 and March 1996, using histological analysis of the ovaries, measurement of gonadosomatic and gonadic indices and the batch fecundity. Five maturity stages for the ovary were described. Mature females were partial spawner because of simultaneous presence of oocytes at different maturity stages. The high frequency of mature females and high gonadosomatic and gonadic indices observed during austral winter and summer suggest two spawning seasons. The estimated batch fecundity (F) for total length (LT) was $F=0.0003 L^{3.9701}$ and for total weight $(\mathrm{PT})$ was $\mathrm{F}=0.2255 \mathrm{PT}^{1.1539}$.
\end{abstract}

Key words: Reproduction, ovary, histology, partial spawner

\section{INTRODUCCIÓN}

El estado del conocimiento de especies de interés para el sector pesquero artesanal en Chile en general es nulo o escaso (Acuña et al. 2007). La cabinza, Isacia conceptionis (Cuvier, 1830), es comercializada por los sectores pesqueros artesanal e industrial (Boré et al. 1988) y se distribuye entre Islas de Lobos de Afuera (Perú) y Corral, Chile (Ojeda 1982, Espino et al. 1989, Chirichigno 1998).

Los mayores desembarques nacionales de la cabinza, en la década de los 90 se produjeron en Coquimbo, con más de 648 ton en 1991 (SERNAPESCA 1990-19991). Entre la información disponible sobre la cabinza hay estudios sobre su pesquería (Espino et al. 1989, Acuña et al. 2007), alimentación de juveniles (Reyes 1992) y parasitofauna asociada (Muñoz 2000). Sobre biología reproductiva, Acuña et al. (2007) describen estados ovocitarios, estados de madurez ovárica, época de desove, fecundidad parcial y talla de madurez sexual para las regiones de Atacama y Coquimbo (Chile). El objetivo del presente trabajo es describir el ciclo reproductivo y fecundidad parcial de Isacia conceptionis en la zona norte de Chile durante un ciclo anual.

\section{MATERIALES Y MÉTODOS}

Entre abril 1995 y marzo 1996, se capturaron mensualmente 30 ejemplares de cabinza mediante pesca con anzuelo (línea

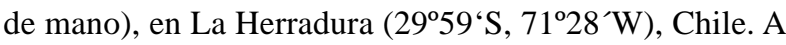
cada ejemplar se le registró la longitud total (LT, cm), peso total (PT, g) y se determinó el sexo. Del total de ejemplares obtenidos ( $\mathrm{n}=360$ ) sólo las hembras fueron analizadas. De las hembras capturadas $(n=280)$ se extrajeron sus ovarios, los que fueron pesados (g) y fijados en formalina al $10 \%$.

El análisis histológico de los ovarios se realizó mediante técnicas tradicionales (Oliva et al. 1986), con cortes transversales de $7 \mu \mathrm{m}$ de las secciones centrales del ovario derecho y teñidos con hematoxilina de Harris y eosina alcohólica para su posterior observación en un microscopio de luz. Los estados ovocitarios se identificaron en las placas histológicas, aplicando los criterios de West (1990) y Tyler \& Sumpter (1996). La madurez ovárica se caracterizó según Oliva et al. (1986) y la tendencia del grado de madurez dentro del ciclo reproductivo se estimó en base a los índices gonadosomático (IGS) (Nikolsky 1963) y gonádico (IG) (Balbontín \& Fischer 1981):

${ }^{1}$ SERNAPESCA. 1990-1999. Anuario Estadístico de Pesca. Ministerio de Economía, Fomento y Reconstrucción, Valparaíso. [en línea]

«ttp:// www. sernapesca.cl> 
IGS $=[$ Peso gónadas $/($ Peso total - Peso gónadas $)] \times 100$

$\mathrm{IG}=\left(\right.$ Peso gónadas $/$ Longitud total $\left.{ }^{3}\right) \times 10000$

El estado fisiológico del pez se estimó con el factor de condición (Arancibia et al. 1994):

$\mathrm{Kc}_{c}=\left[(\right.$ Peso total - Peso gónadas $) /$ Longitud total $\left.^{3}\right] \times 100$

La fecundidad parcial se calculó en 10 hembras maduras, mediante el método gravimétrico (Oliva et al. 1986), en base al recuento de ovocitos maduros (Claramunt et al. 1990, Claramunt et al. 1994).

$$
F=(1 / n) \sum_{n i=1}^{n}\left(H_{i} / W_{n i}\right) \times W_{。}
$$

donde, $\mathrm{F}$ : fecundidad parcial; $\mathrm{H}_{\mathrm{i}}$ : número de ovocitos en submuestra $i ; \mathrm{W}_{\mathrm{ni}}$ : peso submuestra $i$ (g); $\mathrm{W}_{\mathrm{o}}$ : peso ovárico (g) y n: número de submuestras por ovario.

\section{RESULTADOS Y DISCUSIÓN}

Las hembras tuvieron rangos de tamaño corporal de 18-32 cm LT y 130-400 g PT. El análisis histológico de los ovarios permitió reconocer cinco de los seis estados de madurez descritos por Oliva et al. (1986). El ovario en desove, con folículos postovulatorios no se encontró en ninguno de los ejemplares capturados, ni en los estados virginales. Las hembras capturadas en estado inactivo (E1) se presentaron en mayor proporción en marzo y abril, período en el cual la actividad ovárica decreció. El estado previtelogénico (E2) presentó valores altos en los mismos meses que el estado anterior, dando inicio a la madurez ovárica. Los estados vitelogénicos (E3) predominaron en abril- mayo y agostoseptiembre. Las hembras maduras (E4) estuvieron presentes durante todo el año (excepto en abril) y los ovarios hidratados (E5) se registraron sólo en junio-julio y febrero-marzo (Tabla 1).

Las frecuencias mensuales de los ovarios maduros e hidratados indican la existencia de un período de madurez y desove en dos épocas del año, uno principal y de mayor duración entre primavera-verano y uno menor en invierno, períodos que coinciden con altos valores de IGS e IG (Fig. 1) y con lo descrito previamente por Acuña et al. (2007). Los estados E4 y E5 presentan simultáneamente ovocitos en diferentes estados de madurez (Tabla 2), lo que define a la especie como un desovante parcial (Vizziano \& Berois 1990), al igual que la mayoría de los teleósteos de latitudes medias, en donde la actividad reproductiva se extiende durante gran parte del año (Zanuy \& Carrillo 1973, Oliva et al. 1986, Herrera et al. 1988, Chong et al. 1991, Acuña et

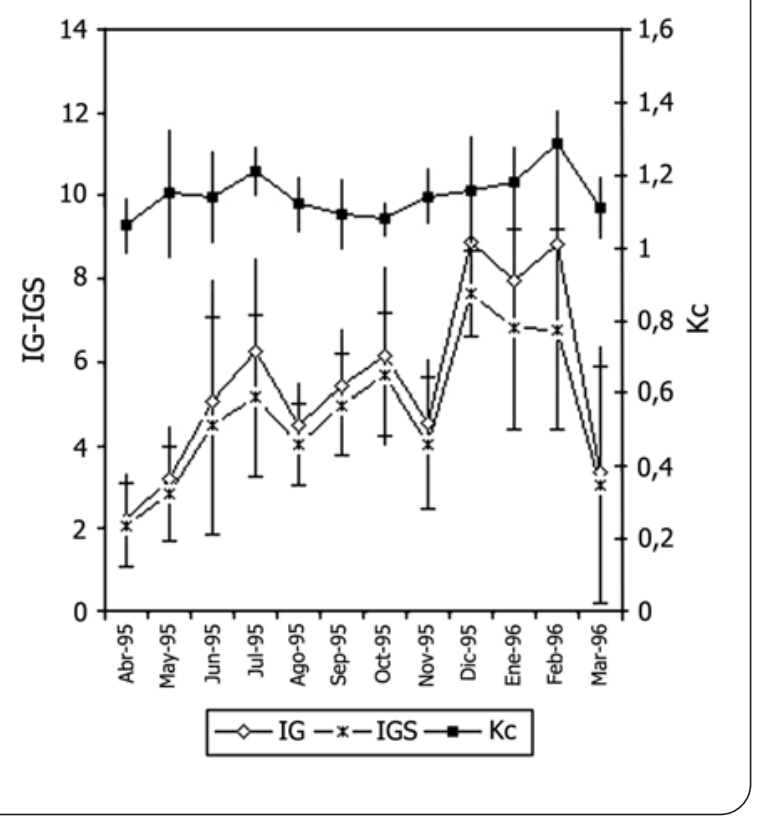

Figura 1. Promedios mensuales y desviación estándar del índice gonádico (IG), índice gonadosomático (IGS) y factor de condición corregido (Kc) para hembras de Isacia conceptionis / Monthly average and standard deviation of gonadal index (IG), gonadosomatic index (IGS) and corrected condition factor (Kc) for Isacia conceptionis females

al. 2007), sin arriesgar el potencial reproductivo en un solo desove (Chong \& González 2009).

La condición fisiológica favorable durante gran parte del año (Kc > 1) para la cabinza (Fig. 1), estaría relacionada con la existencia de una actividad alimentaria permanente en el área, lo cual permite soportar el gasto energético que implica un extenso proceso de maduración y múltiples desoves (Chong et al. 1991, Arancibia et al. 1994, Chong \& González 1995). La Herradura es una bahía donde ocurren eventos de surgencia costera semipermanente de alta productividad primaria, siendo un área óptima de desove y reclutamiento para peces (Acuña et al. 1989).

El modelo que presentó el mejor ajuste entre la fecundidad parcial $(F)$ con el peso corporal $\left(r^{2}=0,798\right)$ y la longitud total $\left(r^{2}=0,797\right)$ es de tipo potencial, quedando expresadas con las funciones: $\mathrm{F}=0,2255 \mathrm{PT}^{1,1539} \mathrm{y} \mathrm{F}=0,0003 \mathrm{LT}^{3,9701}$. La frecuencia del desove y la fecundidad parcial pueden presentar variaciones dentro del ciclo anual, determinadas por la disponibilidad de alimento para el stock desovante, la estructura de edades y la temperatura del mar (Claramunt et al. 1994). Estos factores y la temporalidad de los datos, podrían explicar la baja estimación de la fecundidad parcial 
Tabla 1. Número y frecuencia porcentual mensual de los estados de madurez ovárica de Isacia conceptionis / Number and monthly percent frequency of ovarian maturity stages of Isacia conceptionis

\begin{tabular}{lccrrrrr}
\hline Meses & $\begin{array}{c}\text { Rango longitud } \\
(\mathrm{cm})\end{array}$ & \multicolumn{7}{c}{$\mathrm{N}^{\circ}$} & \multicolumn{5}{c}{ Estados de madurez ovárica } \\
& ejemplares & E1 & E2 & E3 & E4 & E5 \\
\hline 1995 & & & & & & & \\
Abril & $18-24$ & 20 & 38 & 21 & 41 & 0 & 0 \\
Mayo & $21-24$ & 20 & 0 & 2 & 39 & 59 & 0 \\
Junio & $22-28$ & 28 & 0 & 0 & 14 & 65 & 21 \\
Julio & $22-26$ & 23 & 0 & 0 & 20 & 62 & 18 \\
Agosto & $22-27$ & 26 & 0 & 2 & 37 & 61 & 0 \\
Septiembre & $21-27$ & 21 & 0 & 0 & 27 & 73 & 0 \\
Octubre & $22-25$ & 17 & 0 & 0 & 20 & 80 & 0 \\
Noviembre & $22-25$ & 13 & 0 & 0 & 18 & 82 & 0 \\
Diciembre & $23-29$ & 28 & 0 & 0 & 20 & 80 & 0 \\
1996 & & & & & & & \\
Enero & $24-29$ & 28 & 0 & 0 & 15 & 85 & 0 \\
Febrero & $26-32$ & 28 & 0 & 0 & 8 & 87 & 5 \\
Marzo & $24-29$ & 28 & 4 & 15 & 8 & 60 & 13 \\
\hline
\end{tabular}

Tabla 2. Frecuencias porcentuales promedio de los estados ovocitarios presentes en cada estado de madurez ovárica de Isacia conceptionis/ Average percent frequency of oocyte states found in each ovarian maturity stage of Isacia conceptionis

\begin{tabular}{|c|c|c|c|c|c|}
\hline \multirow[b]{2}{*}{ Estados ovocitarios } & \multicolumn{5}{|c|}{ Estados de madurez ovárica } \\
\hline & E1 & E2 & E3 & E4 & E5 \\
\hline Perinucleolar temprano & $99,0 \pm 0,71$ & $37,6 \pm 0,55$ & $16,0 \pm 1,58$ & $6,2 \pm 0,84$ & $15,0 \pm 0,71$ \\
\hline Perinucleolar tardío & & $62,4 \pm 0,55$ & $15,8 \pm 1,48$ & $9,4 \pm 0,89$ & $5,4 \pm 1,40$ \\
\hline Vitelogénico temprano & & & $37,6 \pm 1,67$ & $15,8 \pm 0,45$ & $8,2 \pm 0,45$ \\
\hline Vitelogénico tardio & & & $30,6 \pm 1,14$ & $19,0 \pm 1,00$ & $17,4 \pm 0,55$ \\
\hline Núcleo migrante & & & & $48,2 \pm 1,48$ & $30,8 \pm 0,84$ \\
\hline Hidratado & & & & & $18,4 \pm 1,14$ \\
\hline Atresia & $1,0 \pm 0,82$ & & & $3,5 \pm 0,71$ & $4,8 \pm 0,45$ \\
\hline
\end{tabular}

(51.831 \pm 18.290 huevos promedio) informada por Acuña et al. (2007), a diferencia de lo reportado en el presente trabajo (122.595 \pm 42.452 huevos promedio, estimado en un rango entre los 45.195 y 199.996 huevos por hembra). Esta diferencia en la estimación de la fecundidad parcial podría evidenciar un manejo inadecuado de la explotación de la cabinza en el tiempo, haciendo urgente la necesidad de regular su actividad pesquera.

\section{Agradecimientos}

A la Dra. Karin Lohrman, del Departamento de Biología Marina de la Universidad Católica del Norte, que traspasó sus conocimientos en histología para el desarrollo de la investigación.

\section{LITERATURA CITADA}

Acuña E, J Moraga \& E Uribe. 1989. La zona de Coquimbo: Un sistema de nerítico de surgencia de alta productividad. Revista de la Comisión Permanente del Pacífico Sur, Número Especial: 145-157.

Acuña E, J Villarroel, M Araya, S Hernández, M Andrade, A Cortés \& J Peñailillo. 2007. Estudio biológico-pesquero de los recursos cabinza, machuelo, sierra y blanquillo en la III y IV Regiones. Informe Final Corregido FIP N ${ }^{\circ} 2006-$ 53: 1-247.

Arancibia H, L Cubillos, J Remmaggi \& R Alarcón. 1994. Talla de primera madurez sexual y fecundidad parcial en la sardina común, Strangomera bentincki (Norman, 1936). Biología Pesquera 23: 11-17. 
Balbontín F \& W Fisher. 1981. Ciclo sexual y fecundidad de la merluza, Merluccius gayi gayi, en la costa de Chile. Revista de Biología Marina 17 (3): 285-334.

Boré D, N Henríquez \& G Espinosa. 1988. Chile y sus recursos pesqueros, $69 \mathrm{pp}$. CORFO-IFOP, Santiago.

Claramunt G, G Herrera, I Palape \& E Zagals. 1990. Duración e intensidad del período de desove de 1990 de Sardinops sagax en la zona norte. Informe final. Programa INPESCON 1990, Universidad Arturo Prat, Departamento Ciencias del Mar, Iquique, Chile. Documentos Técnicos 44(1): 1-27.

Claramunt G, G Herrera \& P Pizarro. 1994. Producción potencial anual de huevos por tallas en Sardinops sagax (Jenyns, 1842) del norte de Chile. Revista de Biología Marina 29(2): 211-233.

Chirichigno N. 1998. Clave para identificar los peces marinos del Perú, 496 pp. Instituto del Mar del Perú, Publicación Especial, Callao, Perú.

Chong J \& P González. 1995. Ciclo reproductivo del lenguado de ojos chicos Paralichthys microps (Günther, 1881) (Pleuronectiformes, Paralichthydae) frente al litoral de Concepción, Chile. Biología Pesquera 24: 39-50.

Chong J \& P González. 2009. Ciclo reproductivo y talla media de madurez del congrio colorado, Genypterus chilensis (Guichenot, 1881) en el litoral de Talcahuano, Chile. Revista de Biología Marina y Oceanografía 44(1): 257262.

Chong J, C Oyarzún, E Aguilera \& AArrizaga. 1991. Ciclo de maduración y puesta de la sardina española, Sardinops, sagax (Clupeidae), en la zona pesquera de Talcahuano, Chile (1984-1985). Scientia Marina 55(2): 397-404.

Espino M, M Véliz \& E Valdivia. 1989. Algunos aspectos sobre la biología y pesquería de los recursos que sustentan la actividad artesanal en el Perú, Revista de la Comisión Permanente del Pacífico Sur 18: 49-68.
Herrera G, E Bustos-Obregón \& F Balbontín. 1988. Morphological aspects of gonadal maduration in the hake, Merluccius gayi gayi. Revista de Biología Marina 24(1): 55-71.

Muñoz C. 2000. Descripción preliminar de la fauna de ectoparásitos metazoos presentes en Isacia conceptionis (Perciformes, Haemulidae) en Bahía La Herradura, IV Región, Coquimbo. Evaluación estacional, influencia de la talla y el sexo del hospedador. Tesis de Biología Marina, Universidad Católica del Norte, Coquimbo, 75 pp.

Nikolsky GV. 1963. The ecology of fishes, 352 pp. Academic Press, New York.

Ojeda P. 1982. Iconografía de los principales recursos pesqueros de Chile. I. Zona norte central, 87 pp. Subsecretaría de Pesca, Valparaíso.

Oliva J, W Carvajal \& A Tresierra. 1986. Biología reproductiva e histología de gónadas de peces, 151pp. Editorial Biocience, Trujillo.

Reyes S. 1992. Alimentación de juveniles de Sciaena deliciosa (Tschudii, 1844), Prolatilus jugularis (Valenciennes, 1883) e Isacia conceptionis (Steindachner, 1876) (Pisces, Perciformes) en la bahía de Coquimbo, IV Región, Chile. Tesis de Biólogo Marino, Universidad Católica del Norte, Facultad de Ciencias del Mar, Coquimbo, 61 pp.

Tyler C \& J Sumpter. 1996. Oocyte growth and development in teleosts. Reviews in Fish Biology and Fisheries 6: 287318.

Vizziano D \& N Berois. 1990. Ciclo histológico del ovario de Macrodon ancylodon (Bloch y Schneidar, 1801) (Teleostei: Scianidae). Biología Pesquera 19: 39-47.

West G. 1990. Methods of assessing ovarian development in fishes: a review. Australian Journal of Marine and Freshwater Research 41: 199-222.

Zanuy S \& M Carrillo. 1973. Estudios del ovario de cabrilla Pararcentropristis cabrilla en relación con la ovogénesis. Investigación Pesquera 37(1):147-165.

Recibido el 30 de junio de 2010 y aceptado el 20 de diciembre de 2010 